\title{
Parameterized Model Order Reduction of Delayed Systems using an Interpolation Approach with Amplitude and Frequency Scaling Coefficients
}

\author{
F. Ferranti*, M. Nakhla**, G. Antonini ${ }^{+}$, T. Dhaene*, L. Knockaert*, A. E. Ruehli ${ }^{++}$ \\ *Dept. of Information Technology (INTEC), Internet Based Communication Networks and Services (IBCN), \\ Ghent University-IBBT, Gaston Crommenlaan 8 Bus 201, 9050 Ghent, Belgium \\ Email: francesco.ferranti@ugent.be; tom.dhaene@ugent.be; luc.knockaert@ugent.be. \\ ** Department of Electronics, Carleton University, Ottawa, ON K1S 5B6, Canada \\ Email:msn@doe.carleton.ca \\ +UAq EMC Laboratory, Dipartimento di Ingegneria Elettrica e dell'Informazione, \\ Università degli Studi dell'Aquila, Monteluco di Roio, 67040, L’Aquila, Italy \\ Email: giulio.antonini@univaq.it \\ ${ }^{++}$Missouri University of Science and Technology, Electromagnetic Compatibility Laboratory, Rolla, Missouri, 65409, USA \\ Email: albert.ruehli@gmail.com.
}

\begin{abstract}
When the geometric dimensions become electrically large or signal waveform rise times decrease, time delays must be included in the modeling. We present an innovative PMOR technique for neutral delayed differential systems, which is based on an efficient and reliable combination of univariate model order reduction methods, amplitude and frequency scaling coefficients and positive interpolation schemes. It is able to provide parameterized reduced order models passive by construction over the design space of interest. Pertinent numerical examples validate the proposed PMOR approach.
\end{abstract}

\section{Introduction}

Complex high-speed systems require 3-D electromagnetic (EM) methods $[1,2]$ as analysis and design tools. Large systems of equations are usually generated by the use of these methods and model order reduction (MOR) techniques are crucial to reduce the complexity of EM models and the computational cost of the simulations, while retaining the important physical features of the original system $[3,4]$. Over the last years, the development of methods to build reduced order models (ROMs) of EM systems has been intensively investigated, with applications to interconnects, vias and high-speed packages [5,6].

When signal waveform rise times decrease and the corresponding frequency content increases or the geometric dimensions become electrically large, time delays must be taken into account and included in the modeling. Simply using rational models can result in significant errors and artifacts. Therefore, systems of neutral delayed differential equations (NDDE) [7] becomes fundamental to accurately describe the behavior of such structures.

Traditional MOR techniques perform model reduction only with respect to frequency, but a typical design process includes design space optimization and exploration, and thus it requires repeated simulations for different design parameter values (e.g. geometrical layout or substrate characteristics). Such design activities call for parameterized model order reduction (PMOR) methods that can reduce large systems of equations with respect to frequency and other design parameters.

Over the years, a limited number of PMOR methods for NDDE systems have been developed [8-10]. They are mainly based on multidimensional moment-matching approaches. The structure of such methods may present some computational problems, and the resulting reduced models usually suffer from oversize when the number of moments to match is high, either because high accuracy (order) is required or because the number of parameters is large. The selection of the multidimensional expansion points and the number of multiparameter moments need to be addressed in these methods.

This paper proposes a PMOR method for NDDE systems, which is based on an efficient and reliable combination of univariate model order reduction methods, amplitude and frequency scaling coefficients and positive interpolation schemes. An innovative passive interpolation of reduced systems at an input-output level using amplitude and frequency scaling coefficients is proposed, which considerably enhances the modeling capability of standard positive interpolation schemes [11] and allows to accurately model highly dynamic systems. The proposed method does not have to deal with multiparameter moment computations and related issues. The expansion points are chosen only along the frequency axis using an adaptive algorithm [12]. Under the assumptions that the original NDDE models are passive and the used univariate MOR methods are passivity-preserving, the proposed PMOR method is able to build parameterized reduced models that are passive over the entire design space of interest.

\section{NDDEs}

Let us assume to be interested in generating an admittance representation $\mathbf{Y}(s)$ having output currents $\mathbf{i}_{p}(t)$ under voltage excitation $\mathbf{v}_{p}(t)$. Considering a global number of unknowns is $n_{u}$, we have [12]

$$
\begin{aligned}
\mathbf{C}(\boldsymbol{\tau}) \frac{d \mathbf{x}(t)}{d t} & =-\mathbf{G}(\boldsymbol{\tau}) \mathbf{x}(t)+\mathbf{B u}(t) \\
\mathbf{i}_{p}(t) & =\mathbf{L}^{T} \mathbf{x}(t)
\end{aligned}
$$

where $\mathbf{x}(t) \in \Re^{n_{u} \times 1}$ and $\boldsymbol{\tau} \in \Re^{n_{\tau} \times 1}$ contains all delays. Considering an $n_{p}$-port formulation, where the only sources are the voltage sources at the $n_{p}$-port nodes, $\mathbf{B}=\mathbf{L}$ with $\mathbf{B} \in \Re^{n_{u} \times n_{p}}$. Each delayed entry of matrices $\mathbf{C}(\boldsymbol{\tau})$ and $\mathbf{G}(\boldsymbol{\tau})$ act as a delay operator for the corresponding entry of vector $\mathbf{x}(t)$. Hence, (1) 
can be re-written in the Laplace domain as:

$$
\begin{aligned}
s \mathbf{C}(s) \mathbf{X}(s) & =-\mathbf{G}(s) \mathbf{X}(s)+\mathbf{B V}_{p}(s) \\
\mathbf{I}_{p}(s) & =\mathbf{L}^{T} \mathbf{X}(s) \\
\mathbf{C}(s) & =\mathbf{C}_{0}+\sum_{k=1}^{n_{\tau}} \mathbf{C}_{k} e^{-s \tau_{k}} \\
\mathbf{G}(s) & =\mathbf{G}_{0}+\sum_{k=1}^{n_{\tau}} \mathbf{G}_{k} e^{-s \tau_{k}}
\end{aligned}
$$

which corresponds to the admittance transfer function

$$
\mathbf{Y}(s)=\mathbf{L}^{T}(s \mathbf{C}(s)+\mathbf{G}(s))^{-1} \mathbf{B}
$$

Among all EM methods, the Partial Element Equivalent Circuit (PEEC) method [2] has been found particularly useful for modeling PCBs, interconnects, and power systems. The PEEC method uses a circuit interpretation of the Electric Field Integral Equation (EFIE) and it is especially suitable to problems involving both electromagnetic fields and circuits. To generate the NDDE systems used in the numerical examples, we have used a PEEC formulation which includes delay elements, called $\tau$ PEEC method [7, 12].

\section{Parameterized Model Order Reduction}

Considering the influence of the design parameters $\boldsymbol{g}=$ $\left(g^{(1)}, \ldots, g^{(\mathrm{N})}\right)$ such as layout and substrate features, the formulation (1a)-(1b) becomes

$$
\begin{aligned}
\mathbf{C}(\boldsymbol{\tau}, \boldsymbol{g}) \frac{d \mathbf{x}(t, \boldsymbol{g})}{d t} & =-\mathbf{G}(\boldsymbol{\tau}, \boldsymbol{g}) \mathbf{x}(t, \boldsymbol{g})+\mathbf{B}(\boldsymbol{g}) \mathbf{u}(t)(7 \mathrm{a}) \\
\mathbf{i}_{p}(t, \boldsymbol{g}) & =\mathbf{L}(\boldsymbol{g})^{T} \mathbf{x}(t, \boldsymbol{g})
\end{aligned}
$$

while (6) becomes

$$
\mathbf{Y}(s, \boldsymbol{g})=\mathbf{L}(\boldsymbol{g})^{T}(s \mathbf{C}(s, \boldsymbol{g})+\mathbf{G}(s, \boldsymbol{g}))^{-1} \mathbf{B}(\boldsymbol{g})
$$

In this section, we describe a PMOR algorithm that is able to include, in addition to frequency, $\mathrm{N}$ design parameters $\boldsymbol{g}=$ $\left(g^{(1)}, \ldots, g^{(\mathrm{N})}\right)$ in the reduced order model. The main objective of this PMOR method is to accurately approximate the original scalable system (having a high complexity) with a reduced scalable system (having a low complexity) by capturing the behavior of the original system with respect to frequency and other design parameters.

3.1 $\tau$ ROMs The proposed PMOR technique starts by computing a set of reduced order models of the admittance matrix $\mathbf{Y}(s, \boldsymbol{g})=\mathbf{L}^{T}(\boldsymbol{g})(s \mathbf{C}(s, \boldsymbol{g})+\mathbf{G}(s, \boldsymbol{g}))^{-1} \mathbf{B}(\boldsymbol{g})$, called $\tau$ ROMs, using the MOR method described in [12] for a set of points in the design space, that we call estimation design space grid. The design space $\mathcal{D}(\boldsymbol{g})$ is considered as the parameter space $\mathcal{P}(s, \boldsymbol{g})$ without frequency. The parameter space $\mathcal{P}(s, \boldsymbol{g})$ contains all parameters $(s, \boldsymbol{g})$. If the parameter space is $\mathrm{N}$-dimensional, the design space is ( $\mathrm{N}-1)$-dimensional. Two design space grids are used in the modeling process: an estimation grid and a validation grid. The first grid is utilized to build the $\tau$ ROMs. The second grid is utilized to assess the capability of parameterized reduced models of describing the system under study in a set of points of the design space previously not used for the construction of the $\tau$ ROMs. To clarify the use of these two design space grids, we show in Fig. 1 a possible estimation and validation design space grid in the case of two design parameters $\boldsymbol{g}=\left(g^{(1)}, g^{(2)}\right)$. A $\tau$ ROM is built for each red cross (x) point in the design space. The set of $\tau$ ROMs is further modeled, as explained in what follows, to build a parameterized reduced model that is evaluated and compared with original NDDE models related to the blue circle (o) design space points. We note that these blue circle (o) points are not used for the generation of the $\tau$ ROMs.

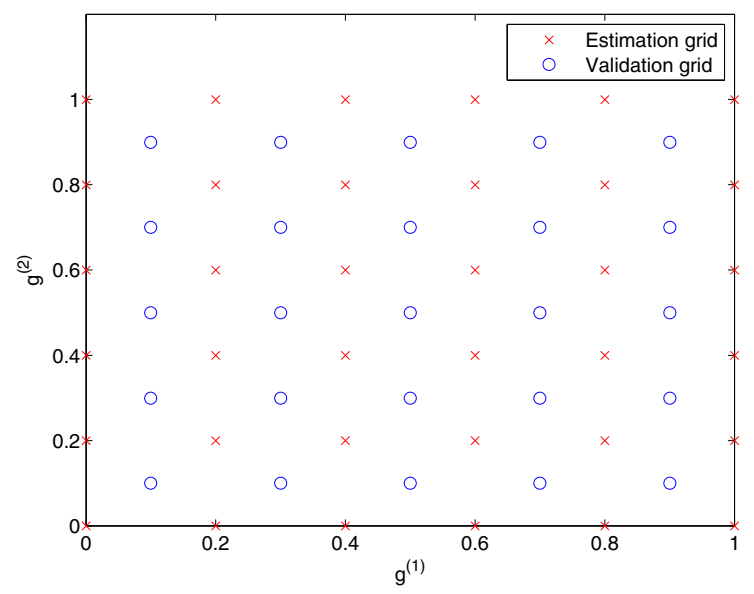

Figure 1: An example of estimation and validation design space grid.

$\mathrm{N}$-dimensional and scattered design space grids can also be treated by the proposed PMOR technique that does not impose any constraint on the number of design parameters and the distribution of $\tau$ ROMs in the design space.

3.2 Amplitude and Frequency Scaling Coefficients After the computation of the $\tau$ ROMs, the next step is building a parameterized $\mathrm{ROM} \mathbf{R}(s, \boldsymbol{g})$. The design space is divided into cells using hyperrectangles (regular grids) [13] or simplices (regular and scattered grids) [14].

Once the design space is divided into cells, a local parameterized model is associated to every cell that is a subdomain of the entire design space. We indicate a cell region of the design space as $\Omega_{i}, i=1, \ldots, P$ and the corresponding vertices as $\boldsymbol{g}_{k}^{\Omega_{i}}, k=1, \ldots, Q$. We note that each vertex corresponds to a $\tau \operatorname{ROM} \mathbf{R}\left(s, \boldsymbol{g}_{k}^{\Omega_{i}}\right)$. For each cell, an optimization procedure is used to find the amplitude and frequency scaling system coefficients that make each vertex an accurate approximant of the other cell vertices. For each vertex $\mathbf{R}\left(s, \boldsymbol{g}_{k}^{\Omega_{i}}\right)$, a set of scaling $\alpha_{1, k}\left(\boldsymbol{g}_{j}^{\Omega_{i}}\right), j=1, \ldots, Q$ and frequency shifting $\alpha_{2, k}\left(\boldsymbol{g}_{j}^{\Omega_{i}}\right), j=1, \ldots, Q$ real coefficients are found, such that

$$
\alpha_{1, k}\left(\boldsymbol{g}_{j}^{\Omega_{i}}\right) \mathbf{R}\left(s \alpha_{2, k}\left(\boldsymbol{g}_{j}^{\Omega_{i}}\right), \boldsymbol{g}_{k}^{\Omega_{i}}\right) \simeq \mathbf{R}\left(s, \boldsymbol{g}_{j}^{\Omega_{i}}\right), j \neq k
$$




$$
\alpha_{1, k}\left(\boldsymbol{g}_{j}^{\Omega_{i}}\right)=\alpha_{2, k}\left(\boldsymbol{g}_{j}^{\Omega_{i}}\right)=1, j=k
$$

The term $\mathbf{R}\left(s, \boldsymbol{g}_{j}^{\Omega_{i}}\right)$ in (9) can be a sampled frequency response based on the original or reduced model at the specific cell vertex. The weighted RMS-error [12] can be used as a cost function to be minimized in the optimization process. If the response of the system under modeling needs to be computed in a specific design space point $\widehat{\boldsymbol{g}}$, a subdomain that contains $\widehat{\boldsymbol{g}}$ is to be found. For each vertex $\tau$ ROM of the found subdomain, the corresponding sets of amplitude and frequency scaling coefficients $\alpha_{1, k}\left(\boldsymbol{g}_{j}^{\Omega_{i}}\right), \alpha_{2, k}\left(\boldsymbol{g}_{j}^{\Omega_{i}}\right)$ are interpolated in $\widehat{\boldsymbol{g}}$ and a model $\widehat{\alpha}_{1, k} \mathbf{R}\left(s \widehat{\alpha}_{2, k}, \boldsymbol{g}_{k}^{\Omega_{i}}\right)$ is built, where $\widehat{\alpha}_{1, k}=\alpha_{1, k}(\widehat{\boldsymbol{g}})$ and $\widehat{\alpha}_{2, k}=\alpha_{2, k}(\widehat{\boldsymbol{g}})$. Finally, the set of modified $\tau$ ROMs $\widehat{\alpha}_{1, k} \mathbf{R}\left(s \widehat{\alpha}_{2, k}, \boldsymbol{g}_{k}^{\Omega_{i}}\right), k=1, \ldots, Q$, can be interpolated at an input/output level as described in [11].

We verified that the use of simple scaling coefficients leads to good results in terms of accuracy, while making the optimization faster and independent from the number of ports. Under the assumptions that the original NDDE models are passive and the MOR method used to provide the $\tau$ ROMs is passivitypreserving, the proposed PMOR method is able to build parameterized reduced models that are passive over the entire design space of interest. We omit the proof due to the lack of space and more details can be found in [15].

\section{Numerical example}

This section presents a numerical example to validate the proposed PMOR approach. The worst case weighted RMSerror [12] over the validation grid is chosen to assess the accuracy and quality of parameterized ROMs.

4.1 Multiconductor system with variable length A multiconductor system has been modeled in this example. The structure is shown in Fig. 2. A bivariate $\tau$ ROM is built as a function of the length $L$ in addition to frequency. Their corresponding ranges are shown in Table 1.

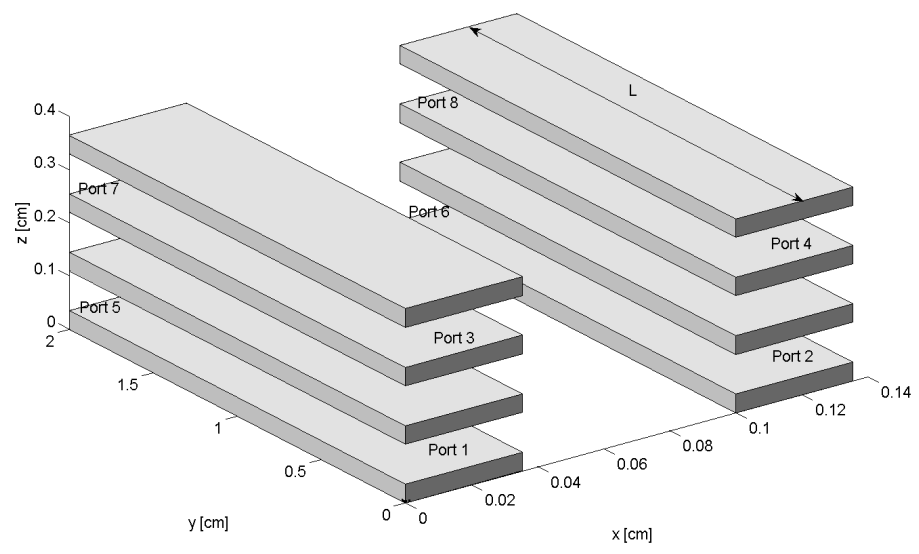

Figure 2: Structure of the multiconductor system.

The $\tau$ PEEC method is used to compute the $\mathbf{C}, \mathbf{G}, \mathbf{B}, \mathbf{L}$ matrices in (1a)-(1b) for 11 values of $L$. Then, we have built reduced models for 6 values of $L$ by means of the MOR algorithm
Table 1: Parameters of the multiconductor system.

\begin{tabular}{|l|c|c|}
\hline Parameter & Min & Max \\
\hline Frequency $($ freq $)$ & $1 \mathrm{~Hz}$ & $15 \mathrm{GHz}$ \\
Length $(L)$ & $1 \mathrm{~cm}$ & $2 \mathrm{~cm}$ \\
\hline
\end{tabular}

described in [12]. Table 2 shows the order and the number of delays of these $6 \tau$ PEEC models and corresponding $\tau$ ROMs.

Table 2: (Order,delays) of $\tau$ PEEC models and $\tau$ ROMs.

\begin{tabular}{|l|c|c|}
\hline & $\tau$ PEEC models & $\tau$ ROMs \\
\hline$L_{1}$ & $(1992,466)$ & $(48,466)$ \\
\hline$L_{2}$ & $(2184,491)$ & $(64,491)$ \\
\hline$L_{3}$ & $(2472,512)$ & $(80,512)$ \\
\hline$L_{4}$ & $(2664,526)$ & $(80,526)$ \\
\hline$L_{5}$ & $(2952,547)$ & $(80,547)$ \\
\hline$L_{6}$ & $(3144,559)$ & $(80,559)$ \\
\hline
\end{tabular}

A bivariate $\tau$ ROM is obtained using the proposed PMOR method with the piecewise linear interpolation scheme. Fig. 3 shows the magnitude of the parameterized reduced model of $\mathbf{Y}_{11}(s, L)$. Fig. 4 shows the magnitude of $\mathbf{Y}_{15}(s, L)$ for the length values $L=\{1.1,1.5,1.9\} \mathrm{cm}$. These specific $L$ values have not been used in the $\tau$ ROMs generation process, nevertheless an excellent agreement between reduced and PEEC models can be observed. The worst case weighted RMS-error is equal to 0.08 . As clearly seen, the parameterized reduced model captures very accurately the behavior of the system.

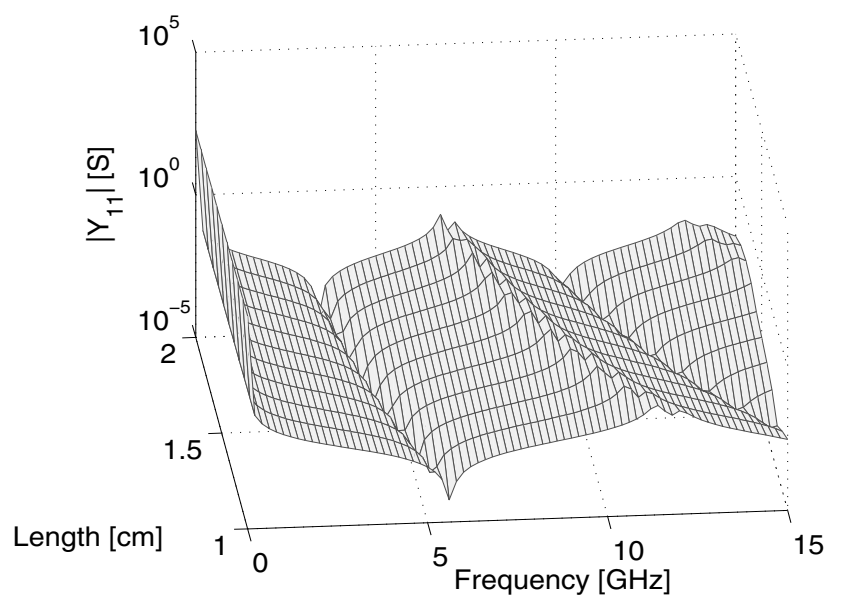

Figure 3: Magnitude of the bivariate $\tau$ ROM of $\mathbf{Y}_{11}(s, L)$.

\section{Conclusions}

We have presented a new PMOR technique applicable to NDDE systems. It is based on an efficient and reliable combination of univariate model order reduction methods, amplitude and frequency scaling coefficients and positive interpola- 


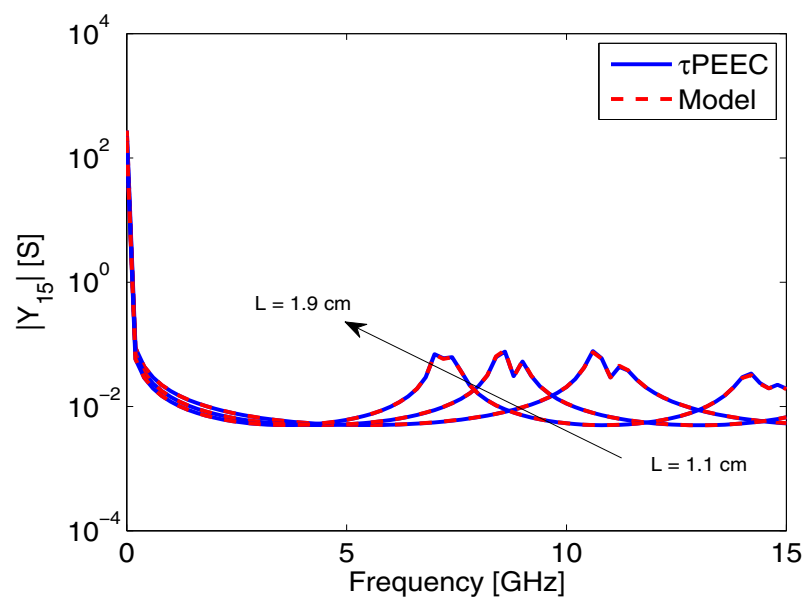

Figure 4: Magnitude of the bivariate $\tau$ ROMs of $\mathbf{Y}_{15}(s, L)$ ( $L=$ $\{1.1,1.5,1.9\} \mathrm{cm})$.

tion schemes. The proposed method does not have to deal with multiparameter moment computation and related issues. The expansion points are chosen only along the frequency axis using an adaptive algorithm. Under the assumptions that the original NDDE models are passive and the used univariate MOR methods are passivity-preserving, the proposed PMOR method is able to build parameterized reduced models that are passive over the entire design space of interest. Numerical results have validated the proposed PMOR approach, showing that it is able to build very accurate parameterized ROMs of dynamic NDDE systems.

\section{References}

[1] R. F. Harrington, Field Computation by Moment Methods. New York: Macmillan, 1968.

[2] A. E. Ruehli, "Equivalent circuit models for three dimensional multiconductor systems," IEEE Trans. Microw. Theory Tech., vol. 22, no. 3, pp. 216-221, Mar. 1974.

[3] P. Feldmann and R. W. Freund, "Efficient linear circuit analysis by Padé approximation via the Lanczos process," IEEE Trans. Comput.-Aided Design Integr. Circuits Syst., vol. 14, no. 5, pp. 639-649, May 1995.

[4] A. Odabasioglu, M. Celik, and L. T. Pileggi, "PRIMA: passive reduced-order interconnect macromodeling algorithm," IEEE Trans. Comput.-Aided Design Integr. Circuits Syst., vol. 17, no. 8, pp. 645-654, Aug. 1998.

[5] R. Achar, M. Nakhla, "Simulation of high-speed interconnects," Proceedings of the IEEE, vol. 89, no. 5, pp. 693728, May 2001.

[6] B. Denecker, F. Olyslager, L. Knockaert, and D. De Zutter, "Generation of FDTD subcell equations by means of reduced order modeling," IEEE Trans. Antennas Propag., vol. 51, no. 8, pp. 1806-1817, Aug. 2003.
[7] P. J. Restle, A. Ruehli, S. G. Walker, G. Papadopoulos, "Full-wave PEEC time-domain for the modeling of on-chip interconnects," IEEE Transactions on ComputerAided Design, vol. 20, no. 7, pp. 877-887, Jul. 2001.

[8] P. Gunupudi, R. Khazaka, and M. Nakhla, "Analysis of transmission line circuits using multidimensional model reduction techniques," IEEE Trans. Adv. Packag., vol. 25, no. 2, pp. 174 - 180, May 2002.

[9] M. Sampath, A. Dounavis, and R. Khazaka, "Parameterized model order reduction techniques for FEM based full wave analysis," IEEE Trans. Adv. Packag., vol. 32, no. 1, pp. 2 -12, Feb. 2009.

[10] M. Ahmadloo and A. Dounavis, "Parameterized model order reduction of electromagnetic systems using multiorder arnoldi," IEEE Trans. Adv. Packag., vol. 33, no. 4, pp. $1012-1020$, Nov. 2010.

[11] F. Ferranti, G. Antonini, T. Dhaene, and L. Knockaert, "Guaranteed passive parameterized model order reduction of the partial element equivalent circuit (PEEC) method," IEEE Transactions on Electromagnetic Compatibility, vol. 52, no. 4, pp. 974 -984, Nov. 2010.

[12] F. Ferranti, M. Nakhla, G. Antonini, T. Dhaene, L. Knockaert, and A. E. Ruehli, "Multipoint full-wave model order reduction for delayed PEEC models with large delays," IEEE Transactions on Electromagnetic Compatibility, vol. 53, no. 4, pp. 959 -967, Nov. 2011.

[13] A.Weiser and S. E. Zarantonello, "A note on piecewise linear and multilinear table interpolation in many dimensions," Mathematics of Computation, vol. 50, no. 181, pp. 189-196, Jan. 1988.

[14] D. F. Watson, "Computing the n-dimensional delaunay tessellation with application to voronoi polytopes," The Computer Journal, vol. 24, no. 2, pp. 167-172, Febr. 1981.

[15] F. Ferranti, M. Nakhla, G. Antonini, T. Dhaene, L. Knockaert, and A. E. Ruehli, "Interpolation-based parameterized model order reduction of delayed systems," IEEE Trans. Microw. Theory Tech., vol. 60, no. 3, pp. 431 - 440, March 2012. 